\title{
DRYING OF A DROP OF POLYMER SOLUTION OF PAN (POLYACRYLONITRILE)-DMF (DIMETHYLFORMAMIDE) SYSTEM
}

\author{
YUJI SANO, SHUICHI YAMAMOTO, HIDEMASA YAMAMOTO \\ AND ROGER B. KEEY \\ Department of Chemical Engineering, Yamaguchi University, Tokiwadai, Ube 755
}

\begin{abstract}
Key Words: Drying, Mass Transfer, Diffusion, Polymer, Polyacrylonitrile, Dimethyl Formamide, Spray Drier

The drying behaviour of a drop of polymer solution of the polyacrylonitrile-dimethylformamide system was investigated in a wide range of drying air temperature (323-423 K), initial drop volume $(2-8 \mu \mathrm{l})$, and initial concentration of solvent in a drop (5-18 kg DMF/ $\mathrm{kg}$ PAN). The theoretical calculation of the drying history of a drop, based on the diffusion equation and the differential heat balance equation, using the experimental values of activity and apparent mutual diffusion coefficient, represented the experimental values satisfactorily. The changes of solvent content and temperature of a drop during drying were well correlated by the ratio (drying time)/(initial radius of a drop) ${ }^{2}$, eliminating the effect of drop size. The shift in controlling step of the drying rate from surface evaporation resistance controlling to internal diffusion resistance controlling is shown by means of changes in the concentrations at the center and the surface of a drop.
\end{abstract}

\section{Introduction}

Drying behaviour of a drop containing dissolved solid is regarded as a fundamental problem in spray drying and has been reported by many investigators. Liquids to be dried by spray driers are classified roughly into three categories. The first are liquids such as an inorganic aqueous solution in which the solid phase appears in a drop in the later stage of drying when the concentration of solid reaches the saturation limit and a crust is formed in the final stage of drying. Charlesworth and Marshal1 ${ }^{1)}$ presented equations to predict the times when the first solid phase appears in a drop and when deposited solid forms a crust. The second category includes a liquid suspensions containing fine particles such as ceramics and minerals which are entirely insoluble in the liquid. As for the drying behaviour of a drop belonging to this category, Ohtani and Miura ${ }^{3)}$ investigated the drying behaviour of a drop containing bentonite particles and showed that the internal resistance of mass transfer is almost negligible. The third are emulsions and colloidal solutions such as a solution containing various food materials or a polymer solution. When a drop of such a solution dries, the internal resistance of mass transfer due to diffusion is usually predominant. A skin is usually formed at the surface after some drying time and wrinkles are

\footnotetext{
Received February 28, 1983. Correspondence concerning this article should be addressed to Y. Sano. H. Yamamoto is now with The Environment Agency, Kasumigaseki, Tokyo, 100. R. B. Keey is at Dept. of Chem. Eng., Canterbury Univ., Christchurch, New Zealand.
}

formed on the surface in the final stage of drying due to shrinkage of the drop. The drying behaviour of the liquid was first analysed by Okazaki et al., ${ }^{2)}$ by solving diffusion equations for the drying of poly(vinyl alcohol) film. The authors presented an analysis of the drying behaviour of a drop of skimmilk aqueous solution, including the effect of inflation of a drop in high-temperature air flow. ${ }^{4)}$

In this report, we also investigate the drying behaviour of a drop of polymer solution of the system PAN (polyacrylonitrile)-DMF (dimethylformamide) for wide ranges of drying conditions, at drying air temperatures below the boiling point of the solvent.

\section{Theoretical Equations}

The authors ${ }^{4)}$ reported a theoretical analysis of drying of a drop of skim milk aqueous solution, taking account of shrinkage due to solvent loss by drying. The same analytical method is applied to the drying of PAN-DMF solution. The following is an outline of the calculation equations.

On assuming a spherically-symmetrical concentration distribution in a drop, a diffusion equation taking account of bulk motion due to diffusion is given by

$$
\frac{\partial \omega_{A}}{\partial t}+\frac{2 D_{A B}}{\omega_{A}+\hat{\omega}}\left(\frac{\partial \omega_{A}}{\partial r}\right)^{2}=\frac{1}{r^{2}} \frac{\partial}{\partial r}\left(r^{2} D_{A B} \frac{\partial \omega_{A}}{\partial r}\right)
$$

Initial and boundary conditions are:

$$
t=0, \quad R=R_{0}, \quad \omega_{A}=\omega_{A 0}, \quad T=T_{0}
$$




$$
\begin{aligned}
r=0, \quad \frac{\partial \omega_{A}}{\partial r} & =0 \\
r=R(t), \quad F & =k_{g}\left(p_{A s}-p_{A}^{*}\right) \\
& =-\rho_{A}{ }^{0} \frac{d R}{d t} \\
& =-\frac{1}{R^{2}} \frac{d}{d t} \int_{0}^{R} \frac{r^{2} \rho_{B}{ }^{0} \hat{\omega}}{\omega_{A}+\hat{\omega}} d r
\end{aligned}
$$

It is assumed that the drop shrinks due to loss of solvent by drying, maintaining a spherical shape. The change of radius of the drop is calculated by Eq. (5).

Assuming uniform temperature in the drop, a heat balance equation yields

$$
\frac{d T}{d t}=\frac{4 \pi R^{2}}{W_{s}} \frac{\left(h_{g}\left(T_{a}-T\right)-F \lambda_{T}\right)}{\left(c_{B}+c_{A} w\right)}
$$

where

$$
w=\frac{4 \pi}{W_{s}} \int_{0}^{R} \frac{r^{2} \rho_{B}^{0} \hat{\omega}}{\omega_{A}+\hat{\omega}} \omega_{A} d r
$$

The heat-transfer coefficient and the mass-transfer coefficient are calculated by the following equations given by Toei and Okazaki. ${ }^{7)}$

$$
\begin{gathered}
N u=2+0.65 \operatorname{Re}^{1 / 2} \operatorname{Pr}^{1 / 3} \\
S h\left(\frac{P_{B m}}{P}\right)^{-0.2}=2+0.65 R e^{1 / 2} S c^{1 / 3}
\end{gathered}
$$

The vapour pressure of solvent at the surface $p_{A s}$ in Eq. (4) is calculated from the activity of the PANDMF system, which was obtained by experiment for the sorption equilibrium of a PAN-DMF film. ${ }^{6}$ The dependency of activity on solvent concentration is shown in Fig. 1. The activity was calculated by a polynomial equation in the drying calculation. The temperature change of the vapour pressure of pure DMF $p_{A}{ }^{0}$ in the relation of $a\left(\omega_{A s}\right)=p_{A s} / p_{A}{ }^{0}$ is given in Table 1.

The mutual diffusion coefficient of the PAN-DMF system measured experimentally by a desorption method of the PAN-DMF films and a microinterferometric method ${ }^{6)}$ were used in the drying calculation by the following equations for a concentration and a temperature considered in the drop.

$$
\begin{aligned}
D_{A B}=\exp \{ & -\frac{28.0+177.5 \omega_{A}}{1+15.0 \omega_{A}} \\
& \left.-\frac{E}{R}\left(\frac{1}{T}-\frac{1}{373.0}\right)\right\} \times 10^{-4}
\end{aligned}
$$

where

$$
\begin{array}{ll}
E=1.38 \times 10^{4} \omega_{A}^{-0.523} & \text { for } \quad \omega_{A}>0.0435 \\
E=7.12 \times 10^{4} & \text { for } \quad \omega_{A} \leqq 0.0435
\end{array}
$$

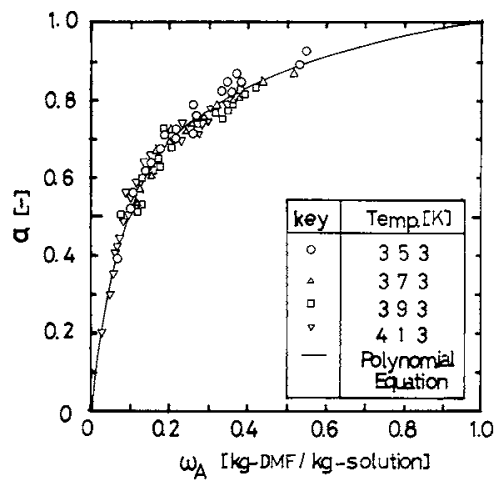

Fig. 1. Activity of PAN-DMF system.

The mutual diffusion coefficient of this system shows an extreme concentration dependency as shown in Fig. 2. The activation energy $E$ also shows the concentration dependency and is approximately presented by Eq. (12).

The calculated solvent content values based on the mutual diffusion coefficient by Eqs. (11) and (12) overestimate the experimental values in the later drying stage, especially in the drying condition of high air temperature, as shown later.

A modification of the mutual diffusion coefficient was considered for the effect of the temperature by the following equation.

$$
D_{A B}^{\prime}=D_{A B} \exp (-661 / T+2.45)
$$

where $D_{A B}$ is the diffusion coefficient given by Eqs. (11) and (12) and $D_{A B}^{\prime}$ is the corrected diffusion coefficient for the drying of a drop. Equation (13) was obtained by comparison of the integral average diffusion coefficient $\bar{D}_{A B}$ calculated by Eqs. (11) and (12) with $\bar{D}_{A B}$ obtained directly by applying the drying rate equation ${ }^{5)}$ to the falling-rate period of the present drying data in which the approximate constancy of $\omega_{A i} \simeq \omega_{A 0}$ and $\omega_{A s} \simeq \omega_{A}{ }^{*}$ were maintained. The drying rate equations, ${ }^{5}$ ) derived by solving the diffusion equation based on the assumption of similar concentration distribution in a drop, are as follows.

$$
\begin{aligned}
F & =\frac{\omega_{A i}-\omega_{A s}}{R_{i}}=\frac{\omega_{A s}-\omega_{A}{ }^{*}}{R_{0}}=\frac{\omega_{A i}-\omega_{A}{ }^{*}}{R_{i}+R_{0}} \\
R_{i} & =\frac{K_{Y}}{\bar{D}_{A B}} \frac{R-\delta}{\rho_{B}{ }^{0}}\left(1+\frac{\omega_{A s}}{\hat{\omega}}\right)^{2} \\
R_{0} & =\frac{1}{k_{g} p_{A}{ }^{0} f} \\
f & =\frac{\left(p_{A s}-p_{A}{ }^{*}\right) / p_{A}{ }^{0}}{\omega_{A s}-\omega_{A}{ }^{*}}
\end{aligned}
$$

$R_{i}$ and $R_{0}$ are the internal resistance and the external resistance for drying rate, respectively. The integral average diffusion coefficient $\bar{D}_{A B}$ is defined by 


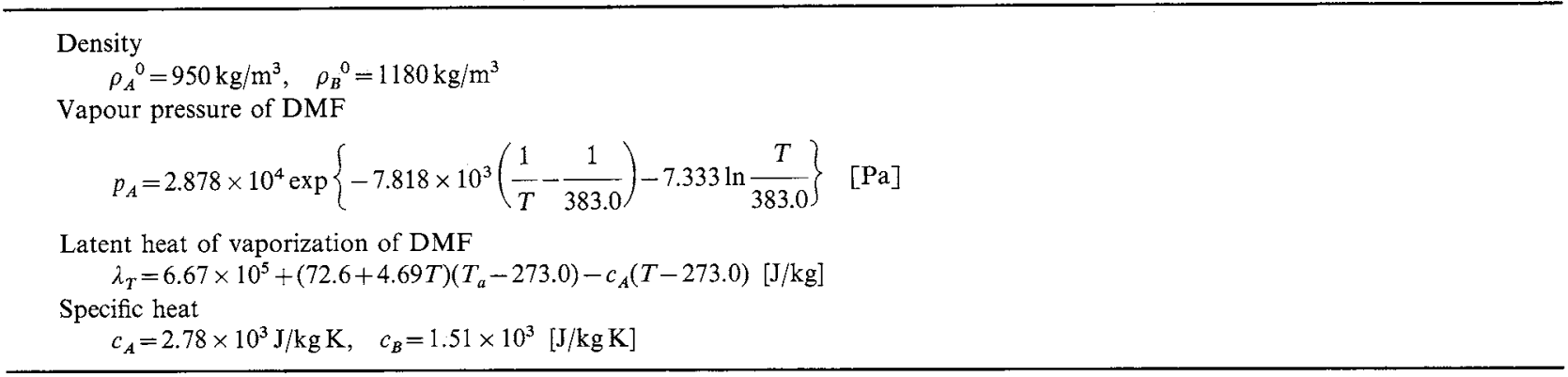

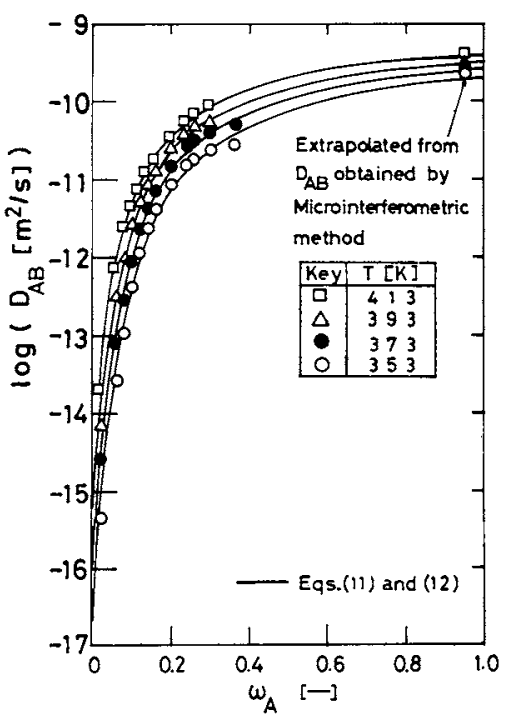

Fig. 2. Mutual diffusion coefficient of PAN-DMF system.

$$
\bar{D}_{A B}=\frac{1}{\omega_{A i}-\omega_{A s}} \int_{\omega_{A s}}^{\omega_{A i}} D_{A B}\left(\omega_{A}\right) d \omega_{A}
$$

Eqs. (14)-(17) are based on the assumption of spherical shrinkage of a drop during drying. In the actual drying, a drop shows an almost spherical shape in the initial stage of drying, but in the later stage the shrinkage due to solvent loss deforms the drop considerably, causing wrinkles in the pliant skin of the drop. This deformation would cause in practice a smaller diffusional distance of the drop in the later stage of drying than that calculated on the assumption of spherical shrinkage of the drop. Then $D_{A B}^{\prime}$ by Eq. (13) would include the above morphological effect and is considered as the apparent diffusion coefficient for the drying of a drop.

For details of the numerical calculation used here, refer to the previous paper. ${ }^{4)}$

\section{Experimental}

The changes in weight and temperature of a drop during drying were measured by the method proposed by Charlesworth and Marshall. ${ }^{1)}$ The experimental apparatus and the procedures were the same as in the previous report. ${ }^{4)}$
The liquid used to form a drop was prepared from PAN and DMF. The polymer of PAN was an acrylonitrile-vinyl acetate copolymer containing $7 \mathrm{wt} \%$ vinyl acetate with a mass average molecular weight of ca. 130,000 .

The physical properties of PAN and DMF are listed in Table 1.

The experiments were arranged for drying conditions that allowed investigation of the effects of the drying air temperature, the initial concentration of the liquid and the initial volume of a drop. The drying air temperature was changed between $323-423 \mathrm{~K}$. Drying when the drying air temperature is higher than the boiling point of DMF (426K) shows some complicated behaviour, and is reported separately elsewhere. The initial concentration was set at the three levels of $\omega_{A 0}=0.85,0.90$ and $0.95 \mathrm{~kg} \mathrm{DMF} / \mathrm{kg}$. The initial volumes of a drop were set at $V_{0}=2,4$ and $8 \mu \mathrm{l}$. The velocity of the drying air stream to a drop was normally kept at a constant value of $1.1 \mathrm{~m} / \mathrm{s}$. As for the experiments to investigate the effect of the drop volume, the air velocity was set at $0.87,1.1$ and $1.4 \mathrm{~m} / \mathrm{s}$ corresponding to the initial drop volume of 8 , 4 and $2 \mu \mathrm{l}$, respectively, in order to examine analogous drying behaviour ${ }^{4)}$ for a constant Reynolds number based on the initial diameter of the drop. In all the experiments, the vapour pressure of the solvent in the drying air stream was zero.

\section{Experimental Results and Discussion}

Typical data of drying history, shown in Fig. 3, were measured at a set of drying conditions of $T_{a}=$ $373 \mathrm{~K}, u_{a}=1.1 \mathrm{~m} / \mathrm{s}, \omega_{A 0}=0.9 \mathrm{~kg} \mathrm{DMF} / \mathrm{kg}\left(w_{0}=9.0 \mathrm{~kg}\right.$ $\mathrm{DMF} / \mathrm{kg} \mathrm{PAN}$ ) and $V_{0}=4 \mu \mathrm{l}$. The data are used as a standard in the following figures for comparison to determine the effects of the drying air temperature, the initial concentration and the initial drop volume. The calculated changes of solvent content $w$ and temperature $T$, based on $D_{A B}$ given by Eqs. (11) and (12), are shown in Fig. 3. The calculated $w$ deviates on the larger side of the experimental values, especially in the later stage of drying. On the other hand, the calculated changes of $w$ and $T$ based on the corrected diffusion coefficient $D_{A B}^{\prime}$ by Eq. (13) are satisfactorily 


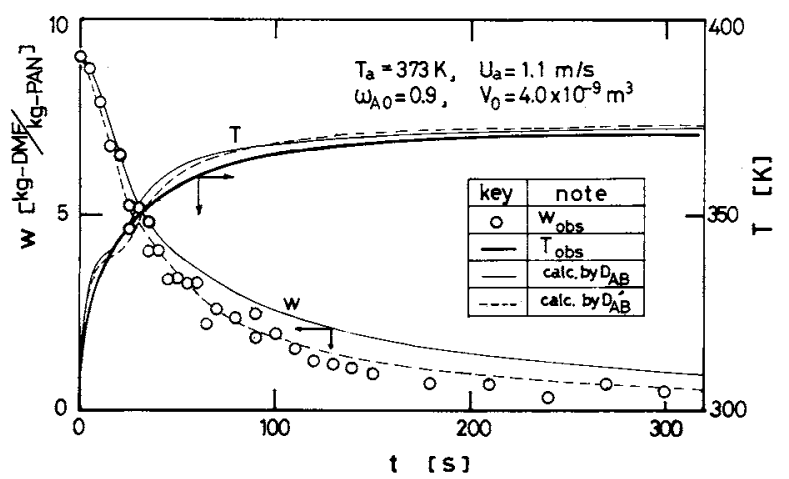

Fig. 3. Sample data of drying history of a drop.

in agreement with the experimental values, as shown in Fig. 3.

The calculated changes of $\omega_{A}$ inside a drop, based on $D_{A B}^{\prime}$ for the data of Fig. 3, are shown in Fig. 4, at the dimensionless radii of $r / R=0\left(\omega_{A i}\right), 0.8,0.98$ and $1.0\left(\omega_{A s}\right)$, respectively. It is noted that the concentration gradients inside a drop are very steep near the surface through the whole drying time, corresponding to the rapid decrease of diffusion coefficient in the lower solvent concentration, as shown in Fig. 2. The activity falls rapidly in the earlier stage of drying, corresponding to the rapid fall of $\omega_{A s}$. No constant-rate period, which may be defined as the drying rate of $a \simeq 1$, exists even in the initial stage of drying, in this data. The ratio of the internal resistance and the total resistance in the drying rate $R_{i} /\left(R_{0}+R_{i}\right)$, calculated with the changes of $\omega_{A i}$ and $\omega_{A s}$ by Eq. (14), is shown in Fig. 4 . The ratio increases rapidly and the internal resistance controls the drying rate entirely after the drying time $80 \mathrm{~s}$. It is pointed out that there is a period between $t=40$ and $120 \mathrm{~s}$ in this data in which the approximate constancies of $\omega_{A i} \simeq \omega_{A 0}$ and $\omega_{A s} \simeq \omega_{A}{ }^{*}=0$ are maintained. In this period, the integral average diffusion coefficient defined by Eq. (18) depends only on the temperature of the drop.

\subsection{The effect of drying air temperature}

The changes of $w$ during drying for the various drying air temperatures are shown in Fig. 5 and the corresponding changes of $T$ are shown in Fig. 6. In these data, the initial concentration $\omega_{A 0}=0.9$, the initial volume of a drop $V_{0}=4 \mu 1$ and the air velocity $u_{a}=1.1 \mathrm{~m} / \mathrm{s}$ were kept constant. The calculated values of $w$ and $T$ under these drying conditions based on $D_{A B}^{\prime}$ are also presented in Figs. 5 and 6, respectively. Agreement between the calculated values based on $D_{A B}^{\prime}$ and the observed ones are satisfactory for the various drying air temperatures while the calculated values of $w$ based on $D_{A B}$ deviate on the larger side from the experimental ones, as shown in Fig. 5.

The characteristic drying curves corresponding to Figs. 5 and 6 are shown in Fig. 7, in which the

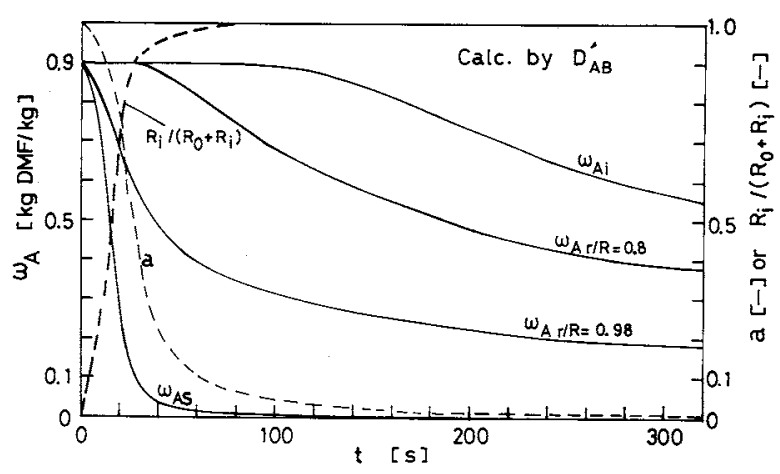

Fig. 4. Changes of $\omega_{A}$ inside a drop, activity of surface and controlling step during drying.

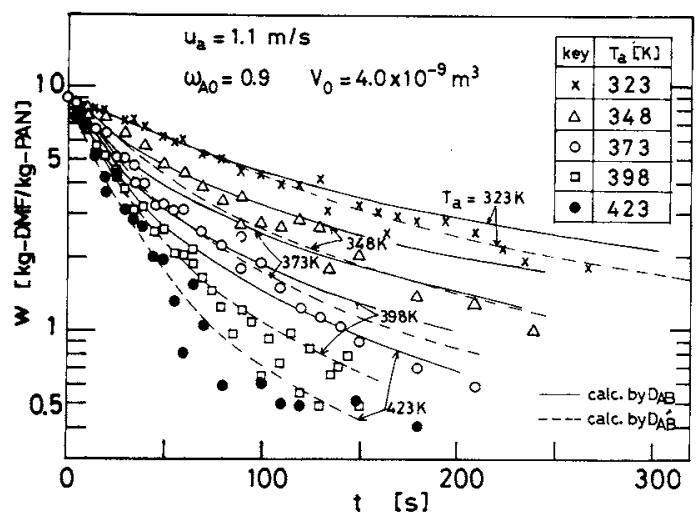

Fig. 5. Changes of solvent content and effect of drying air temperature.

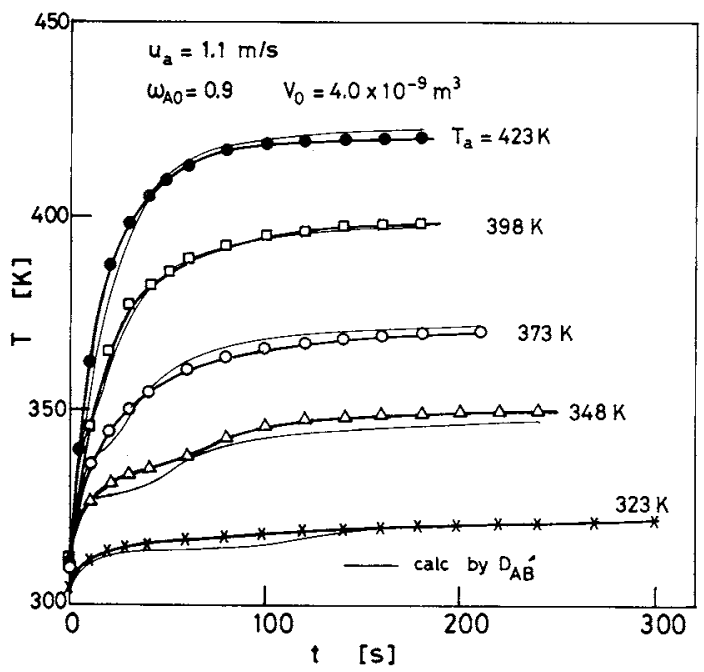

Fig. 6. Changes of drop temperature and effect of drying air temperature.

observed values of the drying rates were obtained by differentiation of the polynomial equations obtained by the application of the least-square method to the observed relations of $w$ vs. $t$. The drying rate curves calculated with $D_{A B}^{\prime}$, shown in Fig. 7 by slender lines, are in agreement with the observed curves shown by 


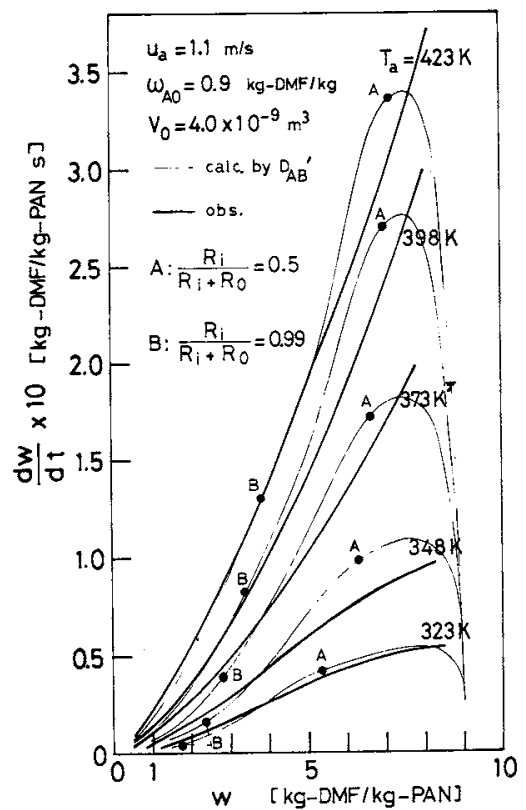

Fig. 7. Characteristic drying curves and effect of drying air temperature.

bold lines. In Fig. 7, point $\mathrm{A}$ denotes the point of $R_{i} /\left(R_{0}+R_{i}\right)=0.5$ and point $\mathrm{B}, R_{i} /\left(R_{0}+R_{i}\right)=0.99$. The manner of the shift of the controlling step according to drying air temperature can be read distinctively on the traces of both points $\mathrm{A}$ and $\mathrm{B}$, as shown in Fig. 7. The higher the drying air temperature, the larger the value of $w$ at which the transition to internal resistance controlling occurs.

\subsection{The effect of initial concentration}

The effect of initial concentration of a drop on drying behaviour is shown in Figs. 8 and 9 for the changes of $w$ and $T$, respectively. The three cases of $\omega_{A 0}=0.85,0.9$ and 0.95 are compared in which $T_{a}=$ $373 \mathrm{~K}, u_{a}=1.1 \mathrm{~m} / \mathrm{s}$ and $V_{0}=4 \mu \mathrm{l}$ were held constant. The calculated values of $w$ based on both $D_{A B}$ and $D_{A B}^{\prime}$ are also shown respectively in Fig. 8. The values of $w$ based on $D_{A B}^{\prime}$ are in agreement with the experimental values while the values based on $D_{A B}$ are considerably higher. The corresponding changes of temperature calculated by $D_{A B}^{\prime}$ are shown in Fig. 9 and are in agreement with the observed changes of $T$.

The characteristic drying curves for the data of Figs. 8 and 9 are shown in Fig. 10. For the case of $\omega_{A 0}=0.95$, the calculated drying rate curve based on $D_{A B}^{\prime}$ shows a rather flat maximum drying rate after the preheating stage, which reflects some external resistance controlling state in the earlier stage of drying. But in the curves for the initially more concentrated liquids of $\omega_{A 0}=0.9$ and 0.85 , the state of the internal resistance controlling comes earlier after the preheating stage.

\subsection{The effect of initial volume of a drop}

From the theoretical point of view, the authors ${ }^{4)}$

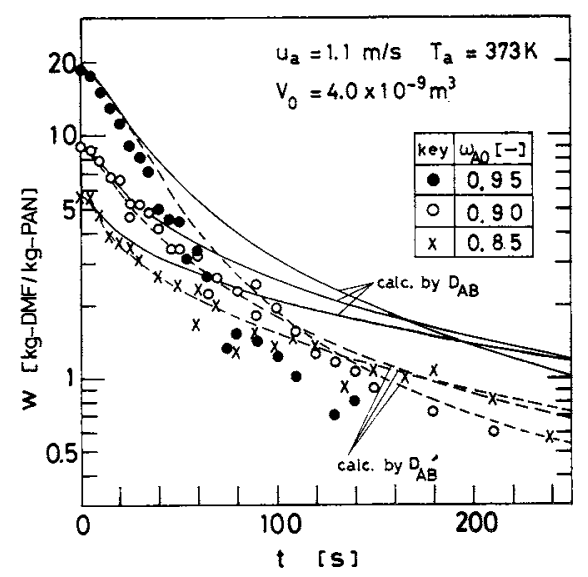

Fig. 8. Changes of solvent content and effect of initial concentration.

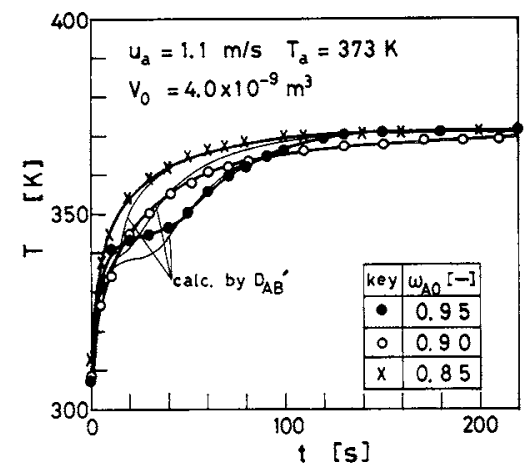

Fig. 9. Changes of drop temperature and effect of initial concentration.

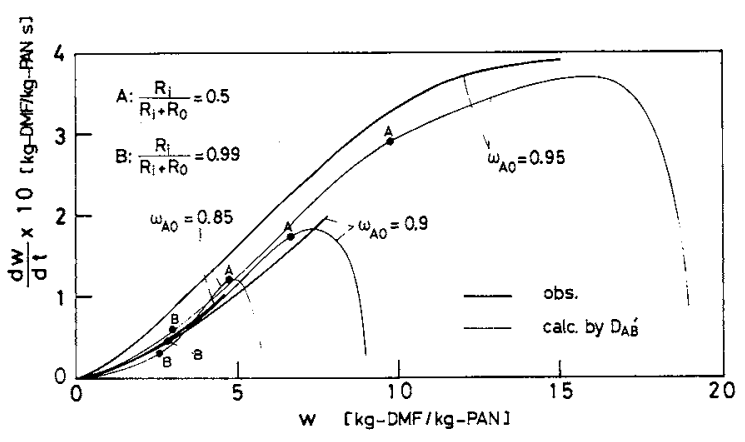

Fig. 10. Characteristic drying curves and effect of initial concentration.

have proposed conditions of similarity for drying of a drop of various sizes. According to this concept, the drying behaviour can be represented similarly by the variable of $t / R_{0}{ }^{2}$ when the Reynolds numbers based on the initial drop diameter are the same. The three cases of initial drop volume of 2,4 and $8 \mu 1$ were tested experimentally. The air velocities were set at $0.87,1.1$ and $1.4 \mathrm{~m} / \mathrm{s}$, respectively, so that the Reynolds numbers based on the initial diameter $2 R_{0}$ were kept constant $\left(R e_{0}=91.6-95.2\right)$. These data are shown in Figs. 11 and 12 for $w$ and $T$, respectively, using the variable $t / R_{0}{ }^{2}$. As shown in Figs. 11 and 12, the use of this variable is quite satisfactory and the changes of $w$ 


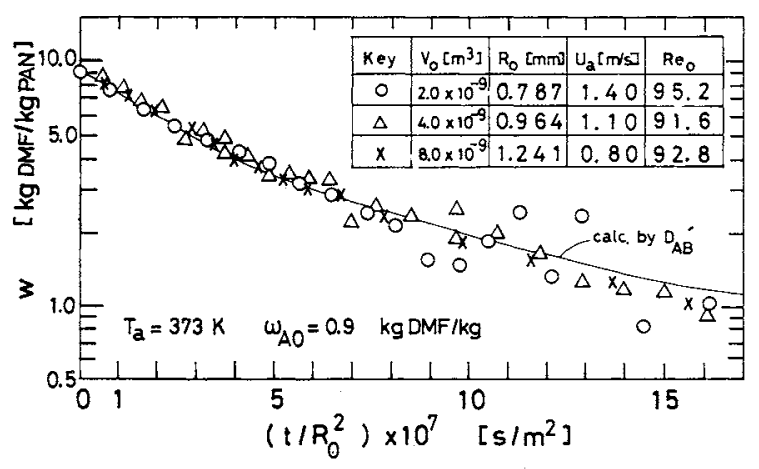

Fig. 11. Changes of solvent content and effect of initial volume of a drop.

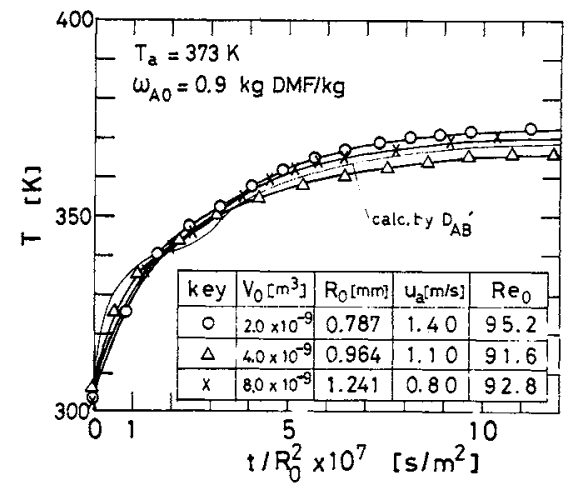

Fig. 12. Changes of drop temperature and effect of initial drop volume.

and $T$ for drops of three different initial radii can be represented by a single relation for both $w$ and $T$, respectively. The calculated curves of $w$ and $T$ based on $D_{A B}^{\prime}$ are in agreement with the experimental data.

\section{Conclusion}

The drying behaviour of a drop of PAN-DMF solution was investigated for various drying conditions. The calculated changes of the solvent content and the temperature of a drop using a corrected apparent diffusion coefficient are in agreement with the experimental results. The changes of solvent content and temperature are well correlated by the term of (drying time)/(initial radius of a drop) $)^{2}$, eliminating the effect of drop size. The shift of the controlling step of the drying rate from external resistance controlling to internal resistance controlling is shown by means of the changes of concentration at the center and the surface of a drop under various drying conditions.

\section{Acknowledgment}

The authors express their thanks to Mitsubishi Rayon Co., Ltd. for its supply of PAN polymer. The assistance of Mr. I. Sinozaki is also appreciated.

\section{Nomenclature}

$\begin{array}{ll}a & =\text { Activity }=p_{A} / p_{A}{ }^{0} \\ c_{A} & =\text { specific heat of solvent }\end{array}$

$\left[\mathrm{J} \mathrm{kg}^{-1} \mathrm{~K}^{[-1}\right]$

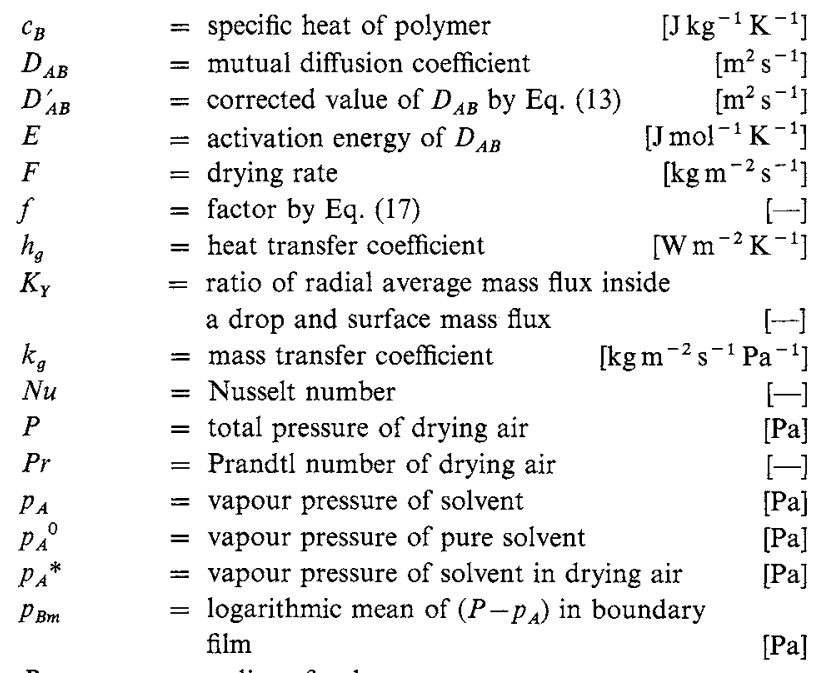

$R \quad=$ radius of a drop or gas constant

$=$ Reynolds number $2 R \cdot u_{d} / v_{a}$

$R_{i} \quad=$ internal resistance for drying rate $\quad\left[\mathrm{m}^{2} \mathrm{~s} \mathrm{~kg}^{-1}\right]$

$R_{0} \quad=$ external resistance for drying rate $\quad\left[\mathrm{m}^{2} \mathrm{~s} \mathrm{~kg}^{-1}\right]$

$r \quad=$ radial distance in a drop $[\mathrm{m}]$

Sc $\quad=$ Schmidt number of solvent vapour in drying air

$=$ Sherwood number

$=$ temperature of a drop

$=$ temperature of drying air

$=$ drying time

$=$ velocity of drying air

$=$ initial volume of a drop

$=$ mass of polymer in a drop

$=$ average solvent content in a drop

$\left[\mathrm{kg}\right.$ solvent $\left.(\mathrm{kg} \text { polymer })^{-1}\right]$

in a drop

$\lambda_{T} \quad=$ latent heat of vaporization $\quad\left[\mathrm{J} \mathrm{kg}^{-1}\right]$

$v_{a} \quad=$ kinematic viscosity of drying air $\quad\left[\mathrm{m}^{2} \mathrm{~s}^{-1}\right]$

$\rho_{A}{ }^{0} \quad=$ density of solvent $\quad\left[\mathrm{kg} \mathrm{m}^{-3}\right]$

$\rho_{B}{ }^{0} \quad=$ density of polymer $\quad\left[\mathrm{kg} \mathrm{m}^{-3}\right]$

$\omega_{A} \quad=$ mass fraction of solvent $\quad\left[\mathrm{kg}\right.$ solvent $\left.\cdot \mathrm{kg}^{-1}\right]$

$\hat{\omega} \quad=\rho_{A}{ }^{0} /\left(\rho_{B}{ }^{0}-\rho_{A}{ }^{0}\right) \quad[-]$

〈Subscripts〉

$i \quad=$ refers to center of a drop

$0 \quad=$ refers to initial state

$s \quad=$ refers to surface of a drop

\section{Literature Cited}

1) Charlesworth, D. H. and W. R. Marshall: AIChE J, 6, 9 (1960).

2) Okazaki, M., K. Shioda, K. Masuda and R. Toei: $J$. Chem. Eng. Japan, 7, 99 (1974).

3) Ohtani, S. and K. Miura: Kagaku Kogaku, 36, 149 (1972).

4) Sano, Y. and R. B. Keey: Chem. Eng. Sci., 37, 881 (1982).

5) Sano, Y. and S. Yamamoto: Proc. 3rd Int. Symposium on Drying, Vol. 1, 535, Birmingham, Sept. (1982).

6) Sano, Y., S. Yamamoto, K. Watanabe and T. Kimura: Kagaku Kogaku Ronbunshu, 9, 1 (1983).

7) Toei, R., M. Okazaki, K. Kubota, K. Ohashi, K. Takaoka and K. Mizuta: Kagaku Kogaku, 30, 43 (1966).

(Presented in part at the Kitakyushu Meeting (Kokura, July, 1981) of The Society of Chemical Engineers, Japan.) 